\title{
Possibilités actuelles des détecteurs ionographiques pour la dosimétrie personnelle des neutrons
}

\author{
A. M. CHAPUIS $(*)$ \\ (Manuscrit reçu le 26 octobre 1976)
}

\begin{abstract}
RÉSUMÉ
Malgré le grand nombre de détecteurs ionographiques préconisés pour la dosimétrie des neutrons très peu sont utilisés, en routine, pour la dosimétrie personnelle. Ceci peut s'expliquer par les nombreux problèmes qui subsistent tant du point de vue théorique que pratique.

L'auteur énumère les difficultés dues à la fabrication, au développement, à la lecture et à l'interprétation de ces dosimètres en donnant quelques valeurs des erreurs introduites par ces paramètres et cite 156 références bibliographiques, classées par sujet.
\end{abstract}

\begin{abstract}
In spite of the large number of solid-state track detectors recommended for neutron dosimetry, a small number are used for routine personal dosimetry. This could be explained by the many problems still extant from both the theoretical and practical points of view. The difficulties due to the manufacture, processing, reading and interpretation of these dosemeters are stated together with some values on the errors introduced by these parameters. 156 references are given, classified by subject.
\end{abstract}

(*) Commissariat à l'Energie atomique, Département de Protection, Service technique d'Études de Protection, BP n 6, 92260 Fontenay-aux-Roses. 
Dès que furent décrites les premières expériences de visualisation des traces de particules lourdes chargées dans certains solides, on assista à un développement rapide des recherches dans ce domaine. Tandis qu'on s'efforçait d'expliquer le phénomène et qu'on découvrait une gamme étendue de nouveaux détecteurs, de nombreuses applications étaient suggérées, étudiées, puis mises en pratique. Elles concernaient des secteurs très différents tels que la biologie, la géologie, la physique nucléaire ou l'étude des rayonnements.

Cette technique ouvrait une nouvelle voie pour la dosimétrie des neutrons. Les possibilités offertes par cette méthode et les caractéristiques des détecteurs permettaient d'envisager rapidement leur utilisation pour la dosimétrie personnelle des neutrons.

Compte tenu du domaine de fluence à mesurer et de l'utilisation, comme convertisseur, de matériaux fissiles en faible quantité, les densités de traces à mesurer étaient très faibles et souvent incompatibles avec un dépouillement au microscope. L'amélioration des techniques de dénombrement était indispensable et donna lieu à la mise au point de nouveaux procédés.

Depuis 1969, l'enthousiasme des premières années étant passé, on cherche à étudier l'influence des divers paramètres, à maîtriser les procédés, à limiter les sources d'erreurs. Les publications insistent alors sur les difficultés et les limites de la méthode. Apparaît en même temps un souci de l' "environnement ", une crainte du risque de contamination et d'irradiation et on observe, dans la plupart des laboratoires, une réticence à faire porter des matériaux fissiles par le personnel. Les recherches s'orientent donc vers un autre type de dosimètre utilisant la détection des particules $\alpha$, des noyaux de recul, des protons. D’autres difficultés apparaissent qui limitent le développement de cette technique.

Le nombre des publications est impressionnant. Beaucoup de dosimètres ont été décrits, mais la plupart sont restés à l'état de prototype. On ne peut être qu'étonné du nombre extrêmement faible de dosimètres personnels effectivement en service dans le monde. Les raisons en sont multiples et nous allons les énumérer brièvement en examinant successivement les dosimètres à convertisseurs fissiles, les dosimètres à particules $\alpha$ ou noyaux de recul et les problèmes liés aux détecteurs, à leur lecture et à l'interprétation des résultats.

\section{DOSIMÈTRES A CONVERTISSEURS FISSILES}

Il existe de nombreux éléments fissiles, mais quatre seulement sont proposés pour la dosimétrie personnelle, les autres ont été rejetés ou abandonnés pour une ou plusieurs des raisons suivantes: période trop courte, section efficace faible, taux de fission spontanée important, activité spécifique élevée, toxicité, fabrication difficile, prix élevé. Les deux derniers critères pourront être modifiés, dans les années à venir, par les progrès réalisés dans le traitement des combustibles irradiés et les recherches sur les transuraniens. Le tableau I donne les caractéristiques principales de ces convertisseurs et quelques exemples d'utilisation. 
TABLEAU I

CARActéristiques de QUelques CONVERTISSEURS Fissiles

\begin{tabular}{|c|c|c|c|c|}
\hline Elément & ${ }^{235} \mathrm{U}$ & ${ }^{238} \mathrm{U}$ & ${ }^{232} \mathrm{Th}$ & ${ }^{237} \mathrm{~Np}$ \\
\hline $\begin{array}{l}\text { Période émission } \alpha \\
\text { Fission spontanée }\end{array}$ & $\begin{array}{l}7.10^{8} \mathrm{a} \\
2.10^{17} \mathrm{a}\end{array}$ & $\begin{array}{l}4,5 \cdot 10^{9} \mathrm{a} \\
6,5 \cdot 10^{15} \mathrm{a}\end{array}$ & $\begin{array}{l}1,4 \cdot 10^{10} \mathrm{a} \\
1,3 \cdot 10^{18} \mathrm{a}\end{array}$ & $\begin{array}{l}2,2 \cdot 10^{6} \mathrm{a} \\
10^{18} \mathrm{a}\end{array}$ \\
\hline $\begin{array}{c}\text { Seuil de la réac- } \\
\text { tion }(n, f) \ldots \ldots \ldots\end{array}$ & & $\sim 1,5 \mathrm{MeV}$ & $\sim 1,5 \mathrm{MeV}$ & $\sim 0,7 \mathrm{MeV}$ \\
\hline 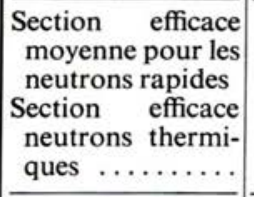 & $\begin{array}{c}1 \mathrm{MeV}: 1,5 \mathrm{~b} \\
600 \mathrm{~b}\end{array}$ & 0,4 à 0,5 b & $0,12 \mathrm{~b}$ & $\begin{array}{l}1,45 \mathrm{~b} \\
19 \mathrm{mb}\end{array}$ \\
\hline $\begin{array}{l}\text { Exemple } \ldots \ldots \ldots \\
\text { Epaisseur } \ldots \ldots \ldots \\
\text { Rendement } \ldots \ldots\end{array}$ & & $\begin{array}{c}\text { Buiss [83] } \\
\text { io0 } \mu \mathrm{m} \\
0.8 \cdot 10^{-5} \text { trace } / n \\
\end{array}$ & $\begin{array}{c}\text { Buiss [83] } \\
50 \mu \mathrm{m} \\
1,4 \cdot 10^{-6} \text { trace } / n\end{array}$ & $\begin{array}{c}\text { Buiss [83] } \\
40 \mu \mathrm{m} \\
1,4 \cdot 10^{-7} \text { trace } / n\end{array}$ \\
\hline $\begin{array}{l}\text { Exemple } \ldots \ldots \ldots \\
\text { Epaisseur ......... } \\
\text { Rendement } \ldots \ldots\end{array}$ & $\begin{array}{c}\text { PRETRE [81] } \\
0,2 \mathrm{~min} \\
\text { alliage } 999 \% \text { Al } \\
1 \% \text { U a } 90 \% \\
0,1 \mathrm{mrad} \\
\text { par étincelle }\end{array}$ & & $\begin{array}{c}\text { PRETRE [81] } \\
20 \mu \mathrm{m} \\
\\
4 \mathrm{mrad} \\
\text { par étincelle }\end{array}$ & \\
\hline
\end{tabular}

\subsection{FABrication du CONVERTISSEUR}

Dans les dosimètres expérimentaux, le convertisseur se présente sous la forme de couche d'épaisseur plus ou moins grande suivant le domaine de dose à mesurer et la facilité de fabrication mais, pour les dosimètres personnels, la quantité de matière fissile doit être réduite au maximum.

Le thorium est utilisé sous forme de film mince de $20 \mu \mathrm{m}[36,81]$ ou de $60 \mu \mathrm{m}$ [83]. L'uranium est obtenu par dépôt électrolytique [67, 80] évaporation $[1,56]$ ou utilisé sous forme d'alliage d'aluminium contenant $1 \mathrm{p}$. mille d'uranium $[36,81]$.

Le neptunium 237, particulièrement intéressant en raison des valeurs du seuil et de la section efficace de réaction de fission, pose des problèmes de fabrication. Ces dépôts, obtenus par électrodéposition ou par évaporation, s'oxydent rapidement et deviennent une source de contamination [145]. On peut recouvrir ce dépôt avec une feuille protectrice en matière plastique qu'il faut manipuler avec soin et contrôler périodiquement [92] ou avec un film de titane pour lequel des essais sont en cours au Centre d'Etudes nucléaires de Fontenay-aux-Roses. On peut également envisager de mélanger le nep- 
tunium avec une résine polyester [5], de le fondre dans des verres [11], de l'incorporer à des céramiques. CROss propose des alliages $\mathrm{Al}-\mathrm{Np}$ contenant 1 p. cent en poids de neptunium ou des mélanges $\mathrm{NpO}_{2}-\mathrm{Au}$ obtenus à partir de poudres, compressées, frittées, puis protégées entre 2 feuilles d'or [145], mais il existe une possibilité de migration du neptunium à travers l'or.

\subsection{CHOIX DU DÉTECTEUR}

Tous les éléments fissiles sont également émetteurs $\alpha$, il faut donc choisir un détecteur dont la sensibilité, pour les conditions d'attaque choisies, ne permette pas la visualisation des particules $\alpha$. Ceci élimine, entre autres, les composés cellulosiques et certains plastiques. On a utilisé avec succès le mica, certains polycarbonates connus sous les noms commerciaux de " Makrofol ", "Lexan » et " Kimfol », et des verres; mais ces derniers sont fragiles et se prêtent mal à la dosimétrie personnelle.

\subsection{IRRADIATION DU PORTEUR}

Lorsque l'élément fissile, ou ses descendants, est émetteur $\beta$ ou $\gamma$, le rayonnement émis peut être suffisant pour irradier le porteur qui doit être protégé du dosimètre lui-même. C'est le cas du neptunium 237 et celui du thorium 232 dont un des descendants, le thorium $C^{\prime}$ émet des photons $\gamma$ de 2,6 MeV. Le dosimètre de Pretre, constitué par un film de thorium de $20 \mu \mathrm{m}$ protégé par $2 \mathrm{~mm}$ d'aluminium, donne un débit de dose au contact d'environ $1 \mathrm{mrem} / \mathrm{h}$ [36]. Le débit de dose au contact est du même ordre de grandeur pour un dosimètre destiné aux doigts comportant une feuille de thorium de $50 \mu \mathrm{m}$ protégée par $0,3 \mathrm{~mm}$ de plomb [82]. Ces protections sont nécessitées par la présence des $\beta$ émis par le bismuth 212 .

L'irradiation produite par ${ }^{237} \mathrm{~Np}$ à $1 \mathrm{~cm}$ est de $1,15 . \mathrm{R} \cdot \mathrm{h}^{-1} \mathrm{par} \mathrm{mCi}$ ou $0,81 \mathrm{mR} \cdot \mathrm{h}^{-1}$ par $\mathrm{mg} ; 75 \mathrm{p}$. cent de ce débit d'exposition est dû aux photons $\gamma$ de 300,312 et $340 \mathrm{keV}$. Pour un organe critique situé à $5 \mathrm{~cm}$ du dosimètre, cela donnerait 57 mrem par an par mg, pour 2000 heures de travail. Le dosimètre décrit par Cross [145] contient $0,6 \mathrm{mg}$ de ${ }^{237} \mathrm{~Np}$; ceci conduit à une dose de 34 mrem par an à $5 \mathrm{~cm}$ et une dose à la peau, à $5 \mathrm{~mm}$ du centre du dosimètre, 40 fois plus forte. Un dosimètre thermoluminescent placé dans le même étui recevrait 15 mrem par semaine.

\section{DOSIMÈTRES A RÉACTIONS $(n, \alpha)$ ET NOYAUX DE RECUL}

L'amélioration de la sensibilité des détecteurs et la découverte de nouvelles matières plastiques sensibles non seulement aux particules $\alpha$ mais également aux deutons et aux protons ont permis d'envisager la dosimétrie des neutrons par l'intermédiaire des particules $\alpha$ et des noyaux de recul. 


\subsection{ChOIX DES DÉTECTEURS}

Ce sont les matériaux les plus sensibles: acétates, nitrates et acétobutyrates de cellulose et certains polycarbonates. Ces plastiques sont fragiles, se rayent facilement et doivent être manipulés avec précaution.

\subsection{ChOIX DES CONVERTISSEURS}

Un premier type de convertisseur est constitué par les composés du bore et du lithium formés à partir des mélanges naturels ou enrichis en bore 10 et en lithium 6. Le convertisseur se présente sous la forme de fluorure de lithium [123, 153], de fluorure de lithium 6 [42, 123], de bore 10 [42], de composés borés comme $\mathrm{B}_{4} \mathrm{C}$, le borax ou l'acide borique [123], de tétraborate de lithium [139].

Les sections efficaces des réactions ${ }^{6} \mathrm{Li}(n, \alpha)$ et ${ }^{10} \mathrm{~B}(n, \alpha)$ pour les neutrons thermiques sont de 955 et 3813 barns ; ces dosimètres sont donc très sensibles. Le dénombrement au microscope optique est utilisable même pour les doses faibles rencontrées en dosimétrie du personnel. Ils permettent de déterminer les fluences de neutrons thermiques autour des réacteurs nucléaires ou en provenance des rayonnements cosmiques [42].

Pour les neutrons d'énergie supérieure à environ $10 \mathrm{MeV}$, on peut mesurer les noyaux de recul de ${ }^{9} \mathrm{Be},{ }^{16} \mathrm{O},{ }^{12} \mathrm{C}$ et ${ }^{13} \mathrm{C}$, les convertisseurs sont alors constitués de film de béryllium, de quartz, de graphite ou de matières plastiques [39, 66, 68].

Il est également possible de détecter les traces en provenance du détecteur lui-même qui joue le rôle de convertisseur. Ce sont donc les réactions sur les éléments constitutifs de la matière plastique et en particulier sur le carbone, l'oxygène et l'azote. Les traces proviennent des particules $\alpha$ émises au cours de réactions $(n, \alpha)$ et des noyaux de recul $[22,35,47,48,75,85$, $118,119,130,134,137,146,151]$. Les réactions sont nombreuses avec des seuils et des sections efficaces différents, les traces ne sont pas différenciables, certaines sont très petites et nous verrons plus loin que cela complique l'interprétation du résultat.

\section{ATTAQUE CHIMIQUE DU DÉTECTEUR}

Les neutrons, par l'intermédiaire du convertisseur, donnent des parti. cules qui laissent des traces dans le détecteur. Ces traces, pour être dénombrées, doivent au préalable être révélées et agrandies par une attaque chimiqueLes caractéristiques de cette attaque ainsi que les conditions dans lesquelles est conservé le détecteur ont une influence sur le nombre de traces, leur forme, leur dimension et donc sur la densité de traces effectivement mesurée. Suivant 
la méthode choisie pour le dénombrement, ces paramètres ont plus ou moins d'importance. Les procédés de lecture qui s'appliquent à des détecteurs épais sont sensibles au diamètre des traces et aux défauts de surface. Ceux qui utilisent des détecteurs sous forme de films minces et dénombrent les traces qui les traversent entièrement sont très sensibles à l'épaisseur du détecteur, à la longueur des traces et à leur orientation.

\subsection{INFLUENCE DU DÉTECTEUR}

Les caractéristiques du détecteur ne sont pas uniquement liées à sa composition chimique. Elles varient avec la technique de fabrication, la nature et la concentration des plastifiants et des stabilisants, les solvants, les conditions de séchage [57]. Pour une même fabrication, il existe des différences d'un lot à l'autre. Par exemple, Congel a noté, en étudiant le rendement de comptage de traces de fragments de fission par la méthode des étincelles, des valeurs qui varient de 82 à 91 p. cent entre deux bains de polycarbonate. Lorsque ces films sont fabriqués spécialement pour les besoins de la dosimétrie, le numéro des lots peut être repéré ; c'est le cas, par exemple, des films de nitrate de cellulose LR 115 de chez Kodak-Pathé. Mais les matières plastiques sont généralement destinées à des usages industriels et produites en grande quantité ; c'est la raison pour laquelle la comparaison des résultats est souvent si difficile. Pour pallier cet inconvénient, la Recherche Coordonnée sur Programme qui, en France, regroupe les scientifiques qui étudient les traces dans les solides a décidé de constituer une banque de détecteurs, réserve où chaque participant peut s'approvisionner et qui assure une homogénéité des caractéristiques et des résultats.

Dans certains cas, la technique de couchage et de séchage du film plastique différencie les deux faces qui ne présentent pas la même sensibilité [110].

\subsection{INFLUENCE DE L'ATTAQue ChimiQue}

Différents paramètres affectent l'attaque chimique. Certains, comme la température, la durée, la concentration initiale, sont facilement contrôlables. Mais on a noté une augmentation de la vitesse d'attaque lorsque le bain alcalin (soude ou potasse) s'enrichit en produits de réaction et en ceux de la dissolution du plastifiant $[57,105]$. C'est cet effet qui se produit, au niveau de la pellicule détectrice, lorsqu'on interrompt l'attaque chimique pour la reprendre ensuite [98, 149], ou lorsqu'on agite la solution [105, 113]. Ces effets ont relativement peu d'importance lorsque le dénombrement est effectué au microscope. Ils ne jouent que sur la valeur de l'énergie minimale nécessaire pour qu'une particule soit détectée, donc sur la détection des $\alpha$ et des noyaux de recul ainsi que sur celle des fragments de fission en provenance d'un convertisseur épais. Par contre, dans le cas du dénombrement par étincelles, la variation de la vitesse d'attaque modifie l'épaisseur du détecteur, donc le rendement de comptage. 


\subsection{INFLUENCE DE L'IRRADIATION}

L'intérêt des détecteurs à traces réside, en particulier, dans le fait qu'ils ne détectent pas les rayonnements $\gamma$, ni les électrons, ni, pour les dosimètres à convertisseurs fissiles, les particules $\alpha$. Mais l'irradiation d'un détecteur à une forte intensité de rayonnement non enregistrable modifie la structure du matériau et augmente la vitesse d'attaque chimique de sa surface.

Ceci entraîne une modification des conditions optimales de visualisation des traces et une diminution du rendement de détection [49, 121, 134]. Ce phénomène peut apparaître pour les dosimètres utilisant un élément fissile d'activité élevée ou ceux portés pendant de longues périodes [92].

\subsection{INFLUENCE DE L'ENVIRONNEMENT}

De nombreuses études portent sur les effets des conditions de stockage avant, pendant et après l'irradiation : influence de la composition de l'atmosphère, de la température, du rayonnement $\gamma$, des rayons ultraviolets... Parmi ces paramètres, les plus importants pour la dosimétrie personnelle sont la température et l'humidité.

On sait qu'un traitement thermique permet d'effacer les traces; ceci est utilisé pour éliminer le bruit de fond des détecteurs, mais on a constaté que le rendement de détection variait avec la durée et la température de ce recuit $[111,112,115]$. Si on utilise ce procédé, il est donc nécessaire de bien en fixer les paramètres et d'effectuer les étalonnages dans les mêmes conditions.

PIESCH a étudié la stabilité des traces de fragments de fission ainsi que celle des particules $\alpha$ et des noyaux de recul dans le Makrofol $E$ et l'acétate de cellulose en fonction de la température et de l'humidité [133]. Pour les $\alpha$ et les noyaux de recul, l'effet est très important dans l'acétate de cellulose, même à température ambiante, lorsque le taux d'humidité dépasse $60 \mathrm{p}$. cent. Dans les conditions du laboratoire, en 3 semaines, 9 p. cent des traces disparaissent.

Pour la même attaque chimique, la présence d'humidité modifie les traces qui sont plus longues et plus larges [60]. BECKER a ainsi noté une augmentation de sensibilité de $50 \mathrm{p}$. cent si le nitrate de cellulose est exposé à l'air au lieu d'être placé dans une enveloppe de papier [35]. Dans ce cas, le phénomène est amplifié par le dénombrement par la méthode des étincelles.

\section{MÉTHODES DE LECTURE}

La première méthode d'examen et de dénombrement des traces fut la vision au microscope optique. Elle reste encore la meilleure lorsqu'il s'agit de mesurer les dimensions des traces, d'étudier leur orientation, de les compter d'une manière précise. Valable encore lorsque la densité de traces n'est pas trop faible et que le nombre de dosimètres est restreint, cette méthode limite le développement de la dosimétrie de routine. 


\subsection{Méthodes optiQues}

Une amélioration possible consiste à augmenter le contraste [30], à utiliser le contraste interférentiel [12, 32] ou à introduire à l'intérieur des traces des produits fluorescents, le dénombrement s'effectuant ensuite sous lumière ultraviolette $[68,105,121]$. La difficulté de cette dernière méthode est due aux défauts de surface qui absorbent également une partie plus ou moins grande des produits.

Une autre technique consiste à augmenter considérablement le diamètre des traces par une attaque chimique en présence d'un champ électrique [64, 134].

L'utilisation d'un système de comptage automatique développé pour les études métallographiques ou le comptage de préparations biologiques a été adaptée au dénombrement des traces : appareils «Quantimet " de Metal Research, "Classimat " de Leitz ou " $\pi$ MC » de Millipore. Il est particulièrement intéressant lorsque la densité de traces est relativement élevée et qu'on désire une bonne précision. Le problème du chevauchement des traces se rencontre peu en dosimétrie personnelle sauf pour les dosimètres à convertisseur de bore et de lithium [46, 71, 88]. L'appareil ne fait pas, comme l'œil, la différence entre les traces et les défauts de surface; or, ceux-ci sont relativement fréquents sur les matières plastiques tendres comme les composés cellulosiques et les polycarbonates.

Dans le film LR 115 de Kodak-Pathé, la surface détectrice très mince et colorée fortement en rouge est sur un support inerte, plus épais et transparent. L'attaque chimique des traces perce la couche colorée et fait apparaître des points blancs sur fond rouge augmentant ainsi nettement le contraste. Il est alors possible soit d'utiliser le système de comptage automatique précédent, soit d'évaluer le nombre total de traces par la surface des trous débouchés en mesurant la lumière transmise par ces trous [102, 128]. Cette méthode s'applique particulièrement bien au cas où les particules sont perpendiculaires à la surface du détecteur. Dans le cas usuel de dosimétrie des neutrons, les $\alpha$ et les noyaux de recul ont des énergies et des orientations très variables. Le résultat est très sensible à l'attaque chimique et à l'épaisseur de la couche colorée et est difficilement reproductible.

En utilisant des matières plastiques transparentes, il est également possible d'évaluer la densité de traces en mesurant la lumière diffusée par la surface. L'attaque chimique est poursuivie pour agrandir le diamètre des traces mais la méthode ne s'applique qu'aux densités élevées $[16,69,70]$.

\subsection{MÉTHOdE ÉleCtriQue}

Pour s'affranchir totalement des mesures optiques, CROss et coll. ont mis au point et expérimenté une méthode de dénombrement par étincelles qui est largement utilisée. Elle consiste à placer le film détecteur mince, après l'attaque chimique, entre deux électrodes dont l'une est constituée par un film plastique aluminisé. Une tension est appliquée entre les deux électrodes, une étincelle 
jaillit entre elles à travers l'un des trous qui traversent le détecteur, cette étincelle volatilise l'aluminium en regard du trou, ce qui limite le claquage à une seule étincelle par trou. En ajustant au mieux les différents paramètres tels que : constantes du circuit électrique (résistance et capacité), haute tension de claquage et de préclaquage, polarité, épaisseur du film détecteur, épaisseur du dépôt d'aluminium, pression exercée sur l'électrode aluminisée et le film détecteur, Cross a obtenu des reproductibilités de 0,5 p. cent.

Dans ces expériences, il s'agissait de traces de fragments de fission normales à la surface et dénombrées, au moment de l'irradiation, à l'aide d'une jonction. Aucune erreur statistique de comptage n'intervenait donc dans la précision. Cette méthode s'applique pour des densités comprises entre une et un millier de traces par $\mathrm{cm}^{2}$ environ.

Le bruit de fond du détecteur vierge, sans tenir compte des traces dues aux fissions spontanées du convertisseur fissile, est de l'ordre de 1 à 2 traces par $\mathrm{cm}^{2}$. Cependant, CARFI [56] en utilisant du Makrofol KG de $10 \mu \mathrm{m}$ d'épaisseur et $19 \mathrm{~mm}$ de diamètre, a trouvé un bruit de fond de

0 trou pour $55 \mathrm{p}$. cent des échantillons,

1 à 5 trous pour 25 p. cent des échantillons,

20 à 50 trous pour $20 \mathrm{p}$. cent des échantillons.

L'examen de ces derniers échantillons a montré que certains étaient légèrement détériorés, ce qui montre l'importance du soin apporté à la manipulation.

Cette méthode est très sensible aux conditions expérimentales et à l'épaisseur du détecteur ; elle s'applique mieux aux dosimètres à convertisseurs fissiles car, d'une part on peut trouver des matériaux détecteurs qui soient minces et plus rigides que les composés cellulosiques et, d'autre part, les traces de fragments de fission sont longues et peuvent facilement traverser le détecteur. Toutefois, en effectuant la dosimétrie auprès d'une source Am-Be, BuRGER estime que la dispersion de ses résultats ( $\sigma=10,5 \mathrm{p}$. cent) est due à des influences non contrôlées sur les taux d'attaque chimique [54].

Dans le cas des traces $\alpha$ et des noyaux de recul, il est nécessaire de poursuivre l'attaque chimique pour que les traces percent le détecteur. Dans ces conditions, la variation du nombre de claquage en fonction de la haute tension appliquée ne présente plus de plateau, que ce soit pour le bruit de fond ou pour les traces dues aux neutrons. Ceci entraîne une diminution de la précision à laquelle vient s'ajouter une variation importante du rendement de détection en fonction de l'énergie des neutrons [146].

La précision de la méthode est également limitée, pour les faibles densités de traces par la statistique de comptage, pour les fortes densités par le chevauchement des zones où l'aluminium est volatilisé. De ce fait, elle ne couvre pas entièrement le domaine de la dosimétrie du personnel et il est nécessaire de superposer au détecteur mince un autre plus épais qui détecte les fragments de fission ayant traversé le premier et qui est lu, au microscope optique, lorsque la densité est trop elevée sur le premier, entre autres, dans le cas d'accidents de criticité [36, 81, 83]. 


\section{INTERPRÉTATION DES RÉSULTATS}

\subsection{INFLUENCE DE L'ÉNERGIE DES NEUTRONS}

La variation de la section efficace des réactions en fonction de l'énergie des neutrons ne permet pas de connaître exactement ni la fluence, ni la dose, si la forme du spectre n'est pas connue. Pour les dosimètres expérimentaux, il est possible de combiner les résultats obtenus avec plusieurs convertisseurs, dont certains sous écran ou sous modérateur, pour approcher d'une manière assez exacte soit la fluence, soit la dose de neutrons et faire même une spectrométrie grossière $[37,38,41,131]$. Ce n'est malheureusement pas le cas des dosimètres personnels pour lesquels le nombre de convertisseurs et le volume sont limités.

Une méthode simple, pour les neutrons rapides, utilise le rapport des sections efficaces de fission des uranium 235 et 238 qui varie avec l'énergie des neutrons ; la combinaison de la réponse de ces deux isotopes peut donc donner une indication sur l'allure du spectre neutronique [46]. Cependant, la grande sensibilité de l'uranium 235 aux neutrons thermiques et des pics de résonance dans la région épithermique peuvent gêner les mesures.

La réponse d'un détecteur au thorium 232 varie d'un facteur 1,5 à 2 entre une source de neutrons de fission du ${ }^{252} \mathrm{Cf}$ et une source Pu-Be [83]. Pretre a déterminé la réponse de son dosimètre au thorium qui est équivalente au rad entre 1,5 et 6,3 MeV ; il existe une hypersensibilité au-dessus de $6,3 \mathrm{MeV}$ qui est d'un facteur 1,3 à $14 \mathrm{MeV}$ et au maximum d'un facteur 4 vers $30 \mathrm{MeV}$ [81].

Les dosimètres au lithium et au bore ont une sensibilité très grande pour les neutrons thermiques. Pour les neutrons épithermiques, la section efficace varie sensiblement comme l'inverse de la vitesse des neutrons. La réponse varie donc avec la forme du spectre. On peut estimer qu'elle est négligeable audessus de $1 \mathrm{keV}$. Entre $0,4 \mathrm{eV}$ et $1 \mathrm{keV}$ cette réponse est environ 15 fois plus faible, pour un spectre en $1 / \mathrm{E}$, que pour les neutrons thermiques [139]. Pour ces mêmes dosimètres, les réactions des neutrons rapides sur le détecteur lui-même donnent naissance à des traces qui peuvent perturber les mesures. Mais les faibles sections efficaces de ces réactions par rapport à celles sur le lithium 6 et le bore 10 ainsi que l'énergie plus faible des particules émises ne rendent ce phénomène gênant que pour les spectres "durs " où neutrons thermiques et épithermiques sont pratiquement absents.

Dans le cas des dosimètres à réaction $(n, \alpha)$ et noyaux de recul, la section efficace des réactions et l'énergie des particules émises varient très rapidement avec l'énergie des neutrons. Ceci ajouté au fait que les particules sont émises à l'intérieur même du détecteur fait que le nombre et la dimension des traces varient énormément avec les conditions de l'attaque chimique et de la méthode de lecture $[85,137]$. Dans son cas particulier, Jozefowicz a déterminé un seuil effectif de mesure d'environ $0,8 \mathrm{MeV}$ et un maximum de réponse entre 2 et $4 \mathrm{MeV}$ [85]. Toutefois, HassiB a montré qu'avec un film spécial (LR 115), la courbe de réponse suit la courbe de dose entre 1,5 et $14 \mathrm{MeV}$ [130]. 


\subsection{INFLUENCE DES NEUTRONS THERMIQUES}

Bien que les réactions de fission de ${ }^{238} \mathrm{U},{ }^{232} \mathrm{Th}$ et ${ }^{237} \mathrm{~Np}$ soient des réactions à seuil, il ne faut pas minimiser l'action des neutrons thermiques sur ces dosimètres. Le neptunium 237 a une section efficace pour les neutrons thermiques de $19 \mathrm{mb}$. Enfin, ils contiennent tous des impuretés, fissiles aux neutrons thermiques, entre autre l'uranium 235 [145].

\subsection{INFLUENCE DU RAYONNEMENT $\gamma$}

C'est le thorium 232 qui est le plus sensible au rayonnement $\gamma$. Il faut éviter de l'utiliser en présence de rayonnement $\gamma$ d'énergie supérieure à $5 \mathrm{MeV}$ pour lequel la réaction de fission $(\gamma, f)$ n'est pas négligeable. De même, il ne faut pas le protéger des neutrons thermiques par du cadmium, ceux-ci donnant sur le cadmium des réactions $(n, \gamma)$ dont les $\gamma$ induisent des fissions dans le thorium [145].

Entre 12 et $15 \mathrm{MeV}$ la section efficace $(\gamma, f)$ pour le ${ }^{237} \mathrm{~Np}$ est environ $200 \mathrm{mb}$ [145].

\subsection{INFLUENCE DE L'ORIENTATION}

Il ne s'agit pas là de l'orientation du porteur du dosimètre par rapport à la source de neutrons (qui est un problème qui se rencontre avec tous les dosimètres personnels pour les neutrons), mais de l'orientation du détecteur luimême par rapport à la direction des neutrons incidents.

L'anisotropie du détecteur à convertisseur fissile est due, en partie, à celle de l'émission des fragments de fission et, en partie, à la prépondérance des fragments de fission légers émis vers l'avant. Ce phénomène est important lorsque le dénombrement est effectué par la méthode des étincelles. L'anisotropie a été mesurée pour ${ }^{232} \mathrm{Th},{ }^{235} \mathrm{U},{ }^{238} \mathrm{U}$ et ${ }^{237} \mathrm{~Np}$ en utilisant du Makrofol $\mathrm{KG}$ de $12 \mu \mathrm{m}$ d'épaisseur et la méthode de comptage par étincelles. L'importance de l'anisotropie dépend de l'attaque chimique et de l'énergie des neutrons. En moyenne, le rapport du nombre de claquages pour deux angles d'incidence de 270 et $180^{\circ}$ est de l'ordre de 1,5 . Entre les directions avant et arrière, on obtient une différence de l'ordre de 25 p. cent [135].

En passant d'une irradiation radiale à une irradiation axiale, Buiss trouve une baisse de rendement qui passe de 58 à 30 pour les sources $(\alpha, n)$ et de 32 à 24 pour les sources de neutrons de fission [83].

Dans le cas des détecteurs à réaction $(n, \alpha)$ et noyaux de recul, l'anisotropie est due à la variation du nombre et de l'énergie des particules en fonction de l'angle d'incidence et de l'énergie des neutrons. Les particules étant issues du détecteur lui-même, l'effet avant-arrière ne doit pas être sensible, par contre il existe une différence entre les irradiations radiales et axiales. 


\subsection{INFLUENCE DE L'ÉPAISSEUR DU CONVERTISSEUR}

La réponse du dosimètre à une fluence donnée de neutrons est fonction de l'épaisseur du convertisseur qui doit donc être déterminée tant que sa valeur est inférieure au parcours maximal des particules à enregistrer.

Les méthodes utilisées: interférence, pesées, mesures de l'activité $\alpha$, renseignent soit sur l'épaisseur en un point, soit sur la quantité totale de matière constituant le convertisseur. Dans l'un et l'autre cas, on suppose que la répartition est uniforme. L'homogénéité de cette répartition est importante lorsque le dénombrement s'effectue au microscope optique sur un nombre restreint de champs ; elle est moins sensible si le dénombrement utilise la presque totalité de la surface du convertisseur.

\subsection{BRUIT DE FOND}

Le bruit de fond qui correspond aux traces enregistrées en l'absence d'irradiation neutronique provient :

- de la fission spontanée des convertisseurs fissiles (PRETRE le trouve égal à 1,4 étincelle par an pour un convertisseur de thorium de $20 \mu \mathrm{m}$ d'épaisseur [81]),

- des émetteurs $\alpha$ au contact avec le détecteur : radon et ses descendants, contamination en uranium...,

- du rayonnement cosmique,

- de défauts enregistrés dans le détecteur ne correspondant pas à des traces réelles, mais dénombrées comme teiles et qui peuvent provenir d'effets mécaniques ou d'actions chimiques entre le convertisseur et le détecteur. Ainsi, les convertisseurs à base de bore et de lithium peuvent dégrader les matières plastiques lorsque l'atmosphère est chaude et humide.

\section{CONCLUSION}

L'énumération des divers paramètres qui influencent la réponse des dosimètres ionographiques ne doit ni décourager, ni d'emblée conduire à l'élimination de cette technique. Au préalable, il est nécessaire de la situer par rapport au dosimètre neutron idéal et par rapport à ce qui existe actuellement. La première comparaison montre qu'il est préférable d'abandonner le terme de « dosimètre " qui sous-entend une mesure de dose, même approchée. La seconde comparaison prouve que toutes les méthodes utilisées actuellement rencontrent des problèmes analogues.

Récemment, un groupe de réflexion du Commissariat à l'énergie atomique sur la dosimétrie individuelle des neutrons a essayé de déterminer les caractéristiques minimales que doit posséder tout dosimètre individuel utilisé en routine. Parmi celles-ci et compte tenu de la difficulté du problème, ce 
groupe a admis que l'erreur commise doit être inférieure à un facteur 2, quel que soit le spectre. Malgré le peu de précision demandée, aucun dosimètre ne convient.

En l'absence d'une mesure de dose, la première obligation est de s'assurer si le personnel est soumis ou non à un champ de neutrons. Dans cette optique, il n'est pas nécessaire d'améliorer inutilement la précision ou d'élaborer des systèmes dosimétriques très compliqués. Par contre, il est indispensable que ces ensembles ne soient pas aveugles à une partie du spectre neutronique. Une évaluation ultérieure de la dose correspondante peut être obtenue en appliquant un coefficient qui tienne compte du spectre réel correspondant. En tant que détecteurs, les systèmes ionographiques retrouvent alors toute leur utilité, à condition de ne pas vouloir leur demander des précisions que, dans l'état actuel des connaissances, ils ne possèdent pas.

\section{BIBLIOGRAPHIE}

\section{Classement des RÉFÉRENCES PAR SUJeT}

Parmi tous les articles sur la détection des particules lourdes chargées par les solides isolants nous avons sélectionné 156 références qui traitent de la dosimétrie des neutrons et des problèmes qui lui sont liés. Afin de faciliter l'exploitation de cette bibliographie nous indiquons ci-dessous les numéros des publications ayant trait à des sujets particuliers.

\section{Dosimètres à convertisseurs fissiles}

Dosimètres expérimentaux : $[1,2,4,6,7,14,15,17,23,37,38,40,41,45,46,54,59,67$, $72,73,86,116,131,132,148]$.

Dosimètres personnels criticité : $[3,18,36,55,72,81]$.

Dosimètres personnels : $[3,11,35,36,56,74,78,80,81,82,92,95,109,145]$.

Dosimètres doigts : $[82,83,125]$.

Détecteur en mica : $[1,14,15,17,59,67,116]$. 148].

Détecteur en makrofol : $[1,6,36,40,46,56.67,72,73,74,80,81,82,83,92,116,131$,

Détecteur en verre : $[1,11,14,15,20]$.

Convertisseur en ${ }^{235} \mathrm{U}:[1,2,4,6,14,17,23,35,36,40,41,46,67,81,87,131]$.

Convertisseur en ${ }^{238} \mathrm{U}:[2,3,4,6,40,41,45,72]$.

Convertisseur en U naturel : $[1,3,11,15,18,20,23,56,67,73,80,86,116]$.

Convertisseur en ${ }^{237} \mathrm{~Np}:[2,3,4,6,40,41,82,92,95,145]$.

Convertisseur en ${ }^{232} \mathrm{Th}:[2,11,15,20,36,40,41,72,73,81,82,83,109]$.

Convertisseur en ${ }^{239} \mathrm{Pu}:[6,40,41,129]$.

Fabrication des convertisseurs : $[1,3,11,17,36,67,80,92,116,126,145]$.

Irradiation due aux convertisseurs : $[36,83,145]$.

Dosimètres à réactions $(n, \alpha)$ et noyaux de recul

Dosimètres expérimentaux : $[22,39,42,65,66,68,75,85,86,103,117,118,119,123,128$, $130,155,156]$.

vOL. $12-\mathrm{N}^{\circ} 2$ 
Dosimètres personnels criticité : $[55,130]$.

Dosimètres personnels : $[35,47,48,134,153]$.

Détection des protons, deutons, tritons : $[19,28,51,97,136]$.

Procédés de lecture

Procédés optiques

Contraste interférentiel : $[12,32]$.

Lumière diffusée : $[16,62,69,70,89]$.

Laser : [120].

Lumière polarisée : [100].

Fluorescence et colorants : $[43,69,100,106,122]$.

Automatisation des lectures : $[10,31,46,94,120]$.

Erreurs de chevauchement : [46, 71, 88].

Microdensitométrie : [155, 156].

\section{Procédés électriques}

Études : $[33,79,87,91,96,107,120]$.

Applications : [35, 36, 48, 50, 56, 59, 67, 73, 80, 81, 87, 92, 131, 140, 146, 147, 148].

Divers : $[3,99,120]$.

\section{Influence de divers paramètres}

Irradiation $\alpha, \beta, \gamma:[43,49,110,112.121,134,145]$.

Température : $[12,24,25,48,105,111,112]$.

Humidité : [24, 25, 48, 52, 60, 92, 105].

Attaque chimique : $[12,25,34,43,46,48,52,57,61,85,98,104,105,106,113,123,143$, $144,146,149]$.

Atmosphère : $[24,25,27,35,43,52,62,105]$.

Orientation : $[35,83,135]$.

Sensibilisation des détecteurs : $[13,27,28,29,44,64,134,142]$.

Articles généraux : [12, 43, 58, 93, 124, 152].

\section{BIBLIOGRAPHIE}

[1] Debeauvais M., Maurette M., Mory J., Walker R. Registration of fission-fragment tracks in several substances and their use in neutron detection. Intern. J. Appl. Radiat. Isot., 1964, 15, 289-299.

[2] MORY J. Sur quelques utilisations de l'enregistrement des fragments de fission dans les isolants. Rapport CEA-R-2846, 1965.

[3] Unruh C. M., Baumgartner W. V., Kocher L. F., Brackenbush L. W., Endres G. W. R. Personnel neutron dosimeter developments. (Rapport BNWL-SA-537) Symposium on neutron monitoring, Vienna 29 Aug.-2 Sept., 1966. Vienne, AIEA, 1976, 433-445.

[4] Baumgartner W. V., Brackenbush L. W., Unruh C. M. A new neutron and high energy particle dosimeter for medical dosimetry applications. Symposium on solid state and chemical radiation dosimetry in medicine and biology, Vienna, Oct. 1966. Vienne, AIEA, 1967, 381-391. 
[5] Baumgartner W. V., BRACKenbush L. W. Rapport BNWL-332, 1966.

[6] KerR G. D., Strickler T. D. The application of solid-state nuclear track detectors for the Hurst threshold detector system. Health Phys., 1966, 12, 1141-1142.

[7] WIDELL C. O. Neutron dosimetry by the fission fragment method. Symposium on neutron monitoring, Vienna, Sept. 1966. Vienne, AIEA, 1967, 417-421.

[8] Chapuis A. M. Applications des méthodes ionographiques à la dosimétrie des neutrons. Radioprotection, 1967 2, 189-194.

[9] TUYN J. W. N. Detection of the tiacks of charged particles in solid materials (en hollandais). Atoomenergie Haar Toepass, 1968, 10, 13-20.

[10] BitTer F., Fiedler G., Wollnik H. Die automatische Auswertung von Kernspur- und Festkörper detektorplatten. Nucl. Instr. Meth., 1967, 51, 241-244.

[11] Yokota R., Kinoshita M., Maruyama M., NishiwaKi Y. Recent improvement of radiophotoluminescent dosimeter and new nuclear track detector. Symposium on Radiation protection monitoring, Bombay 9-13 Dec. 1968, Vienne AIEA, 1969, 287-298.

[12] BENTON E. V. A study of charged particle tracks in cellulose nitrate. Rapport USNRDLTR-68-14, 1968.

[13] Crawford W. T., Desorbo W., Humphrey J. S. Enhancement of track etching rates in charged particle-irradiated plastics by a photo-oxidation effect. Nature, 1968, 220, 13131314.

[14] Maurette M., Mory J. Contribution à la dosimétrie des fluences de neutrons thermiques. Rev. Phys. Appl., 1968, 3, 209-215.

[15] Nishiwaki Y., Tsuruta T., Ono T., Yamazaki K., Fukano Y., Maruyama M. On the detection of neutrons with the etch-pit counting method. Bull. Tokyo Inst. Technol., $1968,83,131-142$.

[16] Schultz W. W. Track density measurement in dielectric track detectors with scattered light. Rev. Scient. Instr., 1968, 39, 1893-1896.

[17] Mourgues M. Application de la technique ionographique à la mesure des flux de neutrons thermiques. Rapport interne SESR/SESRCI-68/90, 1968.

[18] Nicolae M., Pop R. Personal dosimeter for neutrons. Rev. Roum. Phys., 1968, 13, 773 782.

[19] Somogyi G., Varnagy M., Petö G. Application of plastic track detectors for detection of light nuclei. Nucl. Instr. Methods, 1968, 59, 299-304.

[20] Yokota R., Nakajima S., Muto Y. Registration of fission fragment tracks in Th, Au, $\mathrm{U}$ doped phosphate glasses and its possible application to neutron do. imetry. Nucl. Instr. Methods, 1968, 61, 119-120.

[21] à [23] Colloque d'Électronique nucléaire et radioprotection, Toulouse, 4-8 mars 1968.

[21] Debeauvais M., CüER P. Les détecteurs ionographiques dans la dosimétrie et la snectrométrie des neutrons, 3, Session VII, 13 pages.

[22] Stein R., Mosinsky G., Ralarosy J. Dosage de rayonnement et de flux de grande énergie au moyen d'événements enregistrés dans les hauts polymères, 3, Session VII, 15 pages.

[23] Remy G., Tripier J. Spectrométrie des neutrons à l'aide de détecteurs ionographiques solides, 3 , Session VII, 9 pages.

[24] à [42] Journées internationales d'étude de l'enregistrement des traces de particules chargées dans les solides isolants et leurs applications, Clermont-Ferrand, 6-9 mai 1969.

[24] Benton E. V. On latent track formation in organic nuclear charged particle track detectors, 1, chap. 2, 2-27. Radiat. Effects, 1970, 2, 273-280.

[25] Johnson D. R., Boyetr R. H., Becker K. Some studies on the chemical damage mechanism along charged particle tracks in polymers, 1, chap. 2, 46-72.

[26] Beaujean R. Proton tracks in plastic detectors, 1, chap. 2, 114-118.

vOL. $12-\mathrm{N}^{\circ} 2$ 
[27] Desorbo W., Humphrey J. S. Studies on environmental effects upon track etching rates in charged particle-irradiated polycarbonate film, 1, chap. 2, 132-135.

[28] Blanford G. E., Walker R. M., Wefel J. P. Enhancement of track etching by a spark discharge, 1, chap. 2, 95-100; 1, chap. 3, 42-45.

[29] Henke R. P., Benton E, V., Heckman H. H. Sensitivity enhancement of plastic nuclear track detectors through photo-oxidation, 1, chap. 2, 136-147. Radiat. Effects, 1970, 3, 43-49.

[30] MedveczKy L. Track revealing and visualisation, 1, chap. 3, 2-13.

[31] Abmayr W., Grünauer F., Burger G. Automatic optical registration and computer analysis of etched ion-tracks in polymer foils, 1, chap. 3, 46-55.

[32] PIESCH E. Improvements of track visualisation by using an interference contrast method, 1, chap. 3, 66-72.

[33] Cross W. G., Tommasino L. A rapid reading technique for nuclear particle damage tracks in thin foils, 1, chap. 3, 73-85. Radiat. Effects, 1970, 3, 85-89.

[34] Somogyi G., Varnagy M., Medveczky L. The influence of etching parameters on the sensitivity of plastics, 1, chap. 3, 86-99.

[35] BECKER K. Applications of the track etching process in radiation protection, 2, chap. 5, 2-31.

[36] Pretre S. B. Contrôle des doses neutroniques du personnel par le comptage des traces de fragments de fission dans certains plastiques, 2, chap. 5, 41-57.

[37] GrüNauer F., Burger G. Neutron measurements with fission-foil-detectors, 2, chap. 5, 58-71.

[38] Remy G., Ralarosy J., Tripier J., Debeauvais M., Stein R. Dosimétrie et spectrométrie approchées de neutrons de fission et thermonucléaires à l'aide de détecteurs visuels plastiques, 2, chap. 5, 72-83.

[39] Frank A. L., Benton E. V. High energy neutron flux detection with dielectric plastics, 2, chap. 5, 93-102.

[40] Talagrand, Serbat. Mesure de fluences neutroniques par utilisation d'éléments fissiles et de détecteurs solides de traces, 2, chap. 5, 103-113.

[41] Talagrand, Serbat. Spectrométrie neutronique approchée dans l'air à l'aide de détecteurs solides de traces, 2, chap. 5, 114-131.

[42] Roberts J. H., Parker R. A., Congel F. J., Kastner J., Oltman B. G. Environmental neutron measurements with solid state track recorders, 2, chap. 9, 52-55. Radiat. Effects, 1970, 3, 283-285.

[43] MonNin M. Interaction des ions lourds avec des diélectriques organiques macromoléculaires: contribution à l'étude des détecteurs solides de traces. Thèse de doctorat, Clermont-Ferrand, 1969.

[44] Crannell H., Crannell C. J., Kline F. J. Particle track enhancement in cellulose nitrate by application of an electric field. Science, 1969, 166, 606-607.

[45] Nakanishi T., Sakanoue M. Measurement of $14 \mathrm{MeV}$ neutron flux density by fission track method. Radiochem. Radionanal. Letters, 1969, 2, 313-319.

[46] Besant C. B., Ipson S. S. Progress in the application of solid-state track recorders to reactor physics experiments. Rapport AEEW-M-881, 1969.

[47] BECKER K. Nuclear track registration in polymers. Rapport ORNL-4446, 1969, 266.

[48] BECKER K. Alpha particle registration in plastics and its applications for radon and neutron personnel dosimetry. Health Phys., 1969, 16, 113-123.

[49] STONE D. R. Identification of fission fragment tracks in lexan after pre-irradiation to high doses of $\alpha$ particles. Health Phys.. 1969, 16, 772-773. 
[50] Johnson D. R., BOYETT R. H., BeCKeR K. Sensitive automatic counting of alphaparticle tracks in polymers and its applications in dosimetry. Health Phys., 1970, 18, 424-428.

[51] Varnagy M., CsiKai J., Szegedi S., Nagy S. Observation of proton tracks by a plastic detector. Nucl. Instr. Methods, 1970, 89, 27-28.

[52] Boyett R. H., Johnson D. R., Becker K. Some studies on the chemical damage mechanism along charged-particle tracks in polymers. Radiat. Res., 1970, 42, 1-12.

[53] à [56] Symposium Advances in physical and biological radiation detectors, Vienna, 2327 Nov. 1970, Vienne, AIEA, 1971.

[53] GomaA M. A., Hassib G. Neutron dose estimation using a natural uranium fission fragment track personnel dosimeter, 343-347.

[54] Burger G., Grünauer F., Paretzke H. The applicability of track detectors in neutron dosimetry, 349-357.

[55] PIESCH E. Development of new neutron detectors for accident dosimetry, 399-422.

[56] Carfi N., Dugnani Lonati R., Mangialajo M. Réalisation d'un dosimètre pour neutrons utilisant les traces ionographiques dans un solide isolant, 359-364.

[57] VEPRIK Y. M., PERELYGIN V. P. Effect of chemical factors on the sensitivity of polymeric detectors. Prib. Tekh. Eksp., 1970, 4, 51-53. Instrum. Exp. Techn. (USSR) 1970, 4, 1015-1017 (USAEC translation).

[58] BLANC D. Les détecteurs solides de traces. Radioprotection, 1970, 5, 37-78 ; 115-146 ; 201-228.

[59] Congel F. J., Roberts J. H., Armani R. J., Casson H., Gold R., Kastner J., OltMAN B. G. Measurements of absolute thermal neutron densities with mica fission track recorders. Bull. Am. Phys. Soc., 1970, 15, 86.

[60] à [71] $7^{\mathrm{e}}$ Colloque international de photographie corpusculaire et des détecteurs visuels solides, Barcelone, 7-11 juillet 1970 (Cuer, Schmitt, Eds). Strasbourg, Centre de recherches nucléaires.

[60] ENGe W., BeAujean R., Nicken H. P. The influence of humidity on track registration in cellulose nitrate plastic detectors, 1, 175-180.

[61] Peterson D. D. Improvement in particle track etching in lexan polycarbonate film, 1, 181-184.

[62] Barbier J. F. Amélioration des performances du film de nitrate de cellulose utilisé comme enregistreur de traces de particules ionisantes, 1, 187-203.

[63] Chave A., Monnin M. Etude comparée des effets des gaz sur les caractéristiques de détection, 1, 205-210.

[64] Tомmasino L. Electrochemical etching of damage track detectors by use of sinusoidal waveforms, 1, 213-217.

[65] NicolaE M. Enregistrement des traces $\alpha$ dans le nitrate de cellulose et possibilités d'utilisation, 1, 267-274.

[66] Franck A. L., Benton E. V. Measurements of ${ }^{4} \mathrm{He}$ particles and recoil nuclei produced by high energy neutrons in plastics, 2, 441-445.

[67] Congel F. J., Roberts J. H., Armani R. J., Gold R., Kastner J., Oltman B. G. Absolute slow neutron measurements with solid state track recorders, 2, 469-479.

[68] TUYN J. W. N., BRoerse J. J. Analysis of the etch pit size distribution in Makrofol polycarbonate foil after fast neutron exposure, 2, 521-526.

[69] KHAN H. A. Semi-automatic scanning of tracks in plastics, 2, 681-709.

[70] Somogyi G., SRIvastava D. S. Investigations on $\alpha$-ray patterns in dielectric solids, 2, 711-718.

[71] RüEgGer B., Richmond R., ZürCHER W. Les détecteurs solides visuels : comptage automatique des traces et loi de pertes par chevauchement, 2, 723-727. 
[72] Jozefowicz K. Fission track detectors in neutron fluence measurement. Inst. Nucl. Res., Varsaw, Rapport INR-1332, 1971.

[73] Agard E. T., Jervis R. E., McNeil K. G. Neutron dosimetry with nuclear track detectors applied to in vivo neutron activation analysis. Health Phys., 1971, 21, 625-629.

[74] Niкodemova D. New methods in personnel neutron dosimetry. Jaderna Energie, 1971, 17, 312-315.

[75] Nishiwaki Y., Tsuruta T., YamazaKi K. Detection of fast neutrons by etch-pit method of nuclear track registration in plastics. J. Nucl. Sci. Technol., 1971, 8, 162-166.

[76] Cross W. G. Alternatives to nuclear track emulsions for fast neutron personal dosimeters. Health Phys., 1971, 21, p. 4.

[77] GomaA M. A. Calculated response of film badge and fission fragment track dosimeters with indium and cadmium filters to intermediate neutrons. Atomkernenergie, 1971, 18, 321-322.

[78] à [85] Symposium on Neutron monitoring for radiation protection purposes, Vienna, 11-15 Dec. 1972, Vienne, AIEA 1973, 2.

[78] Trousil J., Singer J., Marsal J. Track detectors in the Czechoslovak national personnel dosimetry service, 139-143.

[79] Nishiwaki Y., Kawai H., Morishima H., Koga T., OKada Y. Techniques of spark counting etched nuclear tracks on plastic foils for neutron monitoring, 117-136.

[80] Preston H. E., Peabody C. O. The measurement of personnel neutron dose in reactor and associated areas, 85-97.

[81] PRetre S. Personal neutron dosimeter based on automatic fission-track and spark counting for routine and emergency use, 99-114.

[82] BECKER K. Progress in solid-state fast neutron personnel dosimetry, 145-157.

[83] Buis K., Vaane J. P., Burgkhardt B., Piesch E. Operational experience with a finger dosimeter for fast neutrons, 159-168.

[84] GomaA M. A., Eid A. M., SAYed A. M. Neutron personnel dosimetry with film bagde and fission fragment track dosimeters, 219-225.

[85] Jozefowicz K. Energy dependence of the efficiency of neutron detection in polycarbonate by recording atom recoil tracks, 183-189.

[86] à [90] Meeting of the American Nuclear Society, Washington, Nov. 15-19, 1970. CONF 701102. Trans. Amer. Nucl. Soc., 1970, 13.

[86] TUYN J. W. N. Fast neutron induced etch-pit distributions in Makrofol polycarbonate foil, p. 523.

[87] Congel F. J., Roberts J. H. Automatic system for counting etched holes in thin dielectric plastics, p. 524 et Nucl. Instr. Methods, 1972, 100, 247-252.

[88] Oosterkamp W. J., Schaar J., Van Velze P. L. Automatic counting of solid-state fission track recorders, p. 526.

[89] BARBIER J. Contrast improvement of images obtained in cellulose nitrate film by tracketch methods, p. 530.

[90] BECKER K. Some applications of nuclear track etching in polymers.

[91] Cross W. G., Tommasino L. Improvements in the spark counting technique for damage track neutron dosimeters. Health Phys., 1972, 23, 403.

[92] Sohrabi M., BeCKer K. Fast neutron personnel monitoring by fission fragment registration from ${ }^{237} \mathrm{~Np}$. Nucl. Instr. Methods, 1972, 104, 409-411

[93] BECKER K. Dosimetric applications of track etching. Radiation dosimetry, suppl. 1. (Attix F. H., Ed.), New York, Academic Press, 1972, 79-142.

[94] CoHn C. E., Gold R. Computer-controlled microscope for automatic scanning of solid-state nuclear track recorders. Rev. Sci. Instrum., 1972, 43, 12-17. 
[95] BeCKER K. Applications of track etching in neutron dosimetry. A.N.S. Trans., 1972, 15, (1) p. 116.

[96] Congel F. J., Roberts J. H., Dreis D., Kastner J., Oltman B. G., Gold R., ARMANI R. J. Automatic system for counting etched holes in thin dielectric plastics. Nucl. Instr. Methods, 1972, 100, 247-252.

[97] CARPENTIER B. S., LAFLeUR P. D. Observing proton tracks in cellulose nitrate. Intern. J. Appl. Radiat. Isot., 1972, 23, 157-159.

[98] Baroni G., Di Liberto S., Romano G., Scarbi G., Tabasso M. C. Effect of etching interruptions on tracks formation in plastics. Nucl. Instr. Methods, 1972, 101, 221-227.

[99] Khan H. A., Durrani S. A. Electronic counting and projection of etched tracks in solid state nuclear track detectors. Nucl. Instr. Methods, 1972, 101, 583-587.

[100] MORLey J. A. Two techniques to increase contrast of track etch neutron radiographs. A.N.S. Trans., 1972, 15, (1), p. 120.

[101] Gold R., Armani R. J., Rusch G. K. Fast neutron personnel dosimetry with solidstate track recorders Health Phys., 1972, 23, 404 (P/19).

[102] Chapuis A. M., Gerard N., Soudain G. Nouveau système de lecture pour détecteur ionographique. Radioprotection, 1972, 7, 13-20.

[103] Dragu A., Nicolae M. Application du nitrate de cellulose à la dosimétrie des neutrons rapides. Radioprotection, 1972, 7, 87-102.

[104] Vareille J. C., Teyssier J. L. Un détecteur solide de traces : le triacétate de cellulose. Radioprotection, 1972, 7, 215-228.

[105] VAReille J. C. Etude de la détection des particules chargées par le triacétate de cellulose. Thèse $3^{\mathrm{e}}$ cycle, Université de Limoges, 1972.

[106] Touzery C. Choix et étalonnage de détecteurs plastiques pour l'expérience L.S.C.R.E. Thèse $3^{\mathrm{e}}$ cycle, Université de Clermont-Ferrand, 1972.

[107] à [109] 1st Symposium on neutron dosimetry in biology and medicine, NeuherbergMünchen, 15-19 May 1972. (Rapport EUR-4896 d.f.c.), Luxembourg, Communautés Européennes, 1972.

[107] Cross W. G., Tommasino L. Improvements in the spark counting technique for damage track neutron dosimeters, 283-302.

[108] Fängewisch G. L., Sharmann A. Neuere Ergebnisse bei der Verwendung von Kernspurdetektoren in der Neutronendosimetrie, 303-314.

[109] HeInZELmanN M., SChÜREN H. Thoriumspaltfragmentdosimeter in der Neutronendosimetrie, 315-328. (Dosimètre par fragments de fission du thorium en dosimétrie des neutrons, Traduction C.E.A. 1.624.1).

[110] à [120] 8th International conference on nuclear photography and solid state track detectors, Bucharest 10-15 July, 1972. (Nicolae, Ed.) Bucarest, Institute of atomic physics.

[110] Baroni G., Di Liberto S., Petrera S., Romano G., Sgarbi C. Technical study of the efficiency and of other properties of nitrocellulose detectors, 1, 124-132.

[111] Khan H. A., DURrani S. A. Efficiency calibration of solid state nuclear track detectors 1, 133-151.

[112] Nicolae M. On some factors affecting the sensitivity of plastic track detectors to $\alpha$ particle registration, 1, 178-192.

[113] Bermond J. Cinétique du développement de traces de particules $\alpha$ dans le nitrate de cellulose au cours de traitements continus ou discontinus, 1, 227-234.

[114] SомоGYi G. Effects of ozone atmosphere on the detecting properties of plastic track recorders, 1, 235-252.

[115] SомоGYI G. Influence of thermal effects on the track registration characteristics of plastics, 1, 253-265. 
[116] Danis A., Tommasino L., Oncescu M. Neutron fluence measurements by fission track method using the fissionable element in solutions and glasses, 2, 340-346.

[117] Jozefowicz K. Determination of the energy thresholds for neutron detection in polymer foil track detectors, 2, 347-354.

[118] SPURNY F. Tracks of charged particles formed in polymer foils by fast neutrons : implications to fast neutron dosimetry, 2, 355-361.

[119] Dragu A., Nicolae M. Application du nitrate de cellulose à la dosimétrie des neutrons rapides, 2, 362-377.

[120] Abmayr W., Burger G., Gais P., Paretzke H. G., Platzer H. Automatic measurements of tracks in solid state track detectors with a spark-counter, an on-line flying spot-and TV-device, and with coherent light, 2, 425-432.

[121] Goland A. N., DER MATEOSIAN E. Effect of energetic electron irradiation upon chargedparticle track registration in polycarbonate films. Nucl. Instr. Methods, 1973, 106, 295299.

[122] Paretzke H. G., Gruhn T. A., Benton E. V. The etching of polycarbonate charged particle detectors by aqueous sodium hydroxide. Nucl. Instr. Methods, 1973, 107, 597-600.

[123] Кимамото Y. Alpha tracks in cellulose nitrate as detector elements for spherical moderator type neutron monitors. Health Phys., 1973, 24, 558-559.

[124] BeCKer K. Solid state dosimetry, Cleveland, C. R. C. Press, 1973, 175-224.

[125] MijnheER B. J. Performance of a fission track dosimeter for monitoring the neutron radiation from ${ }^{252} \mathrm{Cf}$ sources on hands inside glove boxes. Rapport EUR-5042, 1973.

[126] Cross W. G., ING H. Preparation and properties of Au-Np radiators for fast neutron damage track personal dosimeters. Rapport AECL-4587, 1973.

[127] à [130] 2nd Symposium on neutron dosimetry in biology and medicine, NeuherbergMünchen, 30 Sept-4 Oct. 1974. (Rapport EUR 3273 d.e.f.) Luxembourg, Communautés européennes, 1.

[127] BECKER K. Some advance in solid-state fast neutron dosimetry, 479-494.

[128] Tripier, J., Oppel R., Remy G., Debeauvais M. Dosimétrie des neutrons rapides par densitométrie, 509-516.

[129] Piesch E., SAYed A. M. Depth dose distribution measurements and attenuation for a ${ }^{252} \mathrm{Cf}$ source, $517-534$.

[130] Hassib G. M., MedveczKy L. New characteristics for cellulose nitrate film as a neutron recoil track detector, 535-544, et Atomki Közlemenyck, 1974, 16, 315-324.

[131] Mijnheer B. J., Pauw H., Van Herk G., Aten A. H. W. Fluence and dose equivalent determination in a neutron field by means of moderating spheres containing fission track recorders. Health Phys., 1973, 24, 423-427.

[132] BIRD T. V., DAvies B. L. An evaluation of the fission track method as a personal neutron dosimeter, Rapport NRPB-R-30, 1974.

[133] PIESCH E., SAYED A. M. Latent fading in solid-state track etching detectors. Nucl. Instr. Methods, 1974, 119, 367-371.

[134] Sohrabi M. The amplification of recoil particle tracks in polymers and its application in fast neutron personnel dosimetry. Health Phys., 1974, 27, 598-600.

[135] MiJNheER B. J. The angular dependence of fast neutron fission track registration. Intern. J. Appl. Radiat. Isot., 1974, 25, 337-345.

[136] Varnagy M., Csikai J., Szegedi S. Effect of electrons and X-rays on alpha-particle registration in T-cellit detector. Nucl. Instr. Methods, 1974, 119, 261-267.

[137] SPURNY Y., Lochmanova J., TUREK K. Dépendance energétique des matériaux plastiques comme détecteurs solides de traces pour les neutrons rapides. Radioprotection, $1974,9,307-324$. 
[138] Rago P. F., Barrall R. C., Carter T. G. A sensitive fast neutron monitor using fission-foil lexan detectors. Health Phys., 1974, 26, 102-103.

[139] ChapuIs A. M. La dosimétrie des neutrons par détecteurs à ionogravure : Possibilités d'applications à la surveillance systématique du personnel. $9^{\text {e }}$ Congrès ATSR sur le contrôle des rayonnements ionisants, Grenoble, 15-18 avril, 1975, 2-16.

[140] JASIAK J., PIFSCH E. Automatical spark counting of neutron-induced recoil tracks in polycarbonate foils. Nucl. Instr. Methods, 1975, 128, 447-454.

[141] Khan H. A. The effects of high gamma doses on the response of plastic track detectors. Nucl. Instr. Methods, 1975, 127, 105-108.

[142] KHAN H. A. Enhancement of the fast-neutron detection efficiency of solid-state nuclear track detectors. Nucl. Instr. Methods, 1975, 126, 557-561.

[143] Enge W., Grabisch K., Dallmeyer L., Bartholomä K. P., Beaujean R. Etching behaviour of the lexan polycarbonate plastic detector. Nucl. Instr. Methods, 1975, 127, 125-135.

[144] Costa-Ribeiro C., Lobao N. Testing of the LR 115 Kodak Pathé red dyed cellulose nitrate for alpha particle detection. Health Phys., 1975, 28, 162-166.

[145] Cross W. G., ING $\mathrm{H}$. The use of ${ }^{237} \mathrm{~Np}$ in personal dosimeters for fast neutrons. Health Phys., 1975, 25, 511-720.

[146] Becker K., ABD el RAZeK M. Automatic spark counting of fast-neutron-induced recoil tracks in polymer foils (ORNL-TM-4460). Nucl. Instr. Methods, 1975, 124, 557561.

[147] BeCKer K. A simple method for the automatic evaluation of particle tracks in mica. Nucl. Instr. Methods, 1975, 126, 553-555.

[148] APt K. E., Schiager K. J. A passive environmental neutron dosimeter. Health Phys., 1975, 28, 474-476.

[149] KHAN H. A. An important precaution in the etching of solid state nuclear track detectors. Nucl. Instr. Methods, 1976, 109, 515-519.

[150] GomaA M. A., EL-Kolaly M. A. Usability of track detectors in mixed field of neutrons and gamma rays. Nucl. Instr. Methods, 1976, 134, 253-256.

[151] Hassib G. M., MedveczKy L. On the origin of the holes observed in LR-115 cellulose nitrate film used for neutron detection. A paraitre.

[152] Fleischer R. L., Price P. V., Walker R. Nuclear tracks in solids, principles and applications. Berkeley University of California press.

[153] Debeauvais M., Tripier J. Etude d'un dosimètre individuel à neutrons. $8^{\mathrm{e}}$ Congrès international de la Société Française de Radioprotection. Aspects fondamentaux et appliqués de la dosimétrie, Saclay, 23-26 mars 1976. Paris, SFRP, 1976, 505-513.

[154] Distenfeld C. H. Composite damage track albedo personnel neutron dosimeter for general use. Rapports BNL-20762, 1975, CONF-760202-25.

[155] HASsiB G. M. Microdensitometric measurements of neutron-induced recoil tracks in plastics and their applications for fast neutron dosimetry. Nucl. Instr. Methods, 1975, 131, 125-128.

[156] HassiB G. M. Some studies on the applications of dielectric track detectors in accidental neutron dosimetry. Atomki, à paraître. 